\title{
Chapter 7 \\ Linking Leaf Spectra to the Plant Tree of Life
}

\author{
José Eduardo Meireles, Brian O’Meara, and Jeannine Cavender-Bares
}

\subsection{Introduction}

Evolution is the engine behind the diversity in leaf structure and chemistry that is captured in their spectral profiles, and, therefore, leaf spectra are inexorably linked to the tree of life. Our ability to distinguish species using spectra is a consequence of trait differences that arise and accumulate over evolutionary time. By the same token, the amount of variation that exists in different spectral regions is ultimately determined by the pace of evolution, convergence, and other evolutionary dynamics affecting the underlying leaf traits. There is an increasing interest in understanding leaf spectra through the lens of evolution and in the context of phylogenetic history (Cavender-Bares et al. 2016; McManus et al. 2016). Advances on this front will require, however, a good understanding of how evolutionary biologists leverage the tree of life to make inferences about evolution.

\footnotetext{
J. E. Meireles $(\bowtie)$

Department of Ecology, Evolution and Behavior, University of Minnesota, Saint Paul, MN, USA

School of Biology \& Ecology, University of Maine, Orono, ME, USA

B. O'Meara

Department of Ecology \& Evolutionary Biology, University of Tennessee, Knoxville, TN, USA

J. Cavender-Bares

Department of Ecology, Evolution and Behavior, University of Minnesota,

Saint Paul, MN, USA 


\subsection{Evolutionary Trees}

We refer to phylogenies in many different ways, and all of these terms appear in the literature. The terms phylogeny, phylogenetic tree, and evolutionary tree can be used interchangeably. We also use the term tree of life to refer to the tree of life (the evolutionary tree for all of life) or to the phylogeny of a really large group (or lineage) of organisms, such as the plant tree of life or the vertebrate tree of life.

\subsubsection{How to Read Phylogenies}

The idea that species descend from a common ancestor is at the very core of the theory of evolution. Evolutionary trees represent the branching structure of life and describe how species are related to each other similarly to how a genealogical tree recounts how people are related. A branch on a phylogenetic tree is a species; when it speciates, two (typically) descendant species arise. The two lineages coming from the same ancestor are known as sisters. These lineages can continue to branch, leading to more descendants. An ancestor and all its descendants are known as a clade: since these descendants all came from the same species, they share many inherited traits. Relatedness among organisms is encoded in the phylogeny's structure-its topology - which defines a series of lineages that are hierarchically nested. The branch lengths also usually convey information, such as the time since divergence, amount of molecular similarity, or number of generations (Fig. 7.1). Dated fossil information can be used to calibrate the age of some of the nodes in a phylogeny. This is one means by which branch lengths can be made to represent time fairly accurately. Typically, the spacing between tip nodes (the $y$-axis in Fig. 7.1a, b, and d) has no meaning, but it can sometimes be used to display information about the trait values of a species (Fig. 7.1c). Because no one has been taking notes of how lineages split over the last 4 billion years, phylogenetic trees must be estimated by analyzing current species data, generally DNA sequences, using models of evolution. This means that phylogenies are statistical inferences that have uncertainty about their topology and their branch lengths (Fig. 7.1d).

\subsubsection{Why Care About Phylogenetic Accuracy?}

An accurate phylogeny is key for understanding life. A phylogeny in which dandelions were more closely related to ferns than to roses would tell us a very different story about the evolution of flowering plants than would the true phylogeny, in which all flowering plants belong to a single lineage. In other words, the accuracy of the estimated tree topology - the structure of the relationships between speciesmatters to how we understand trait evolution. Accurately inferring divergence times 


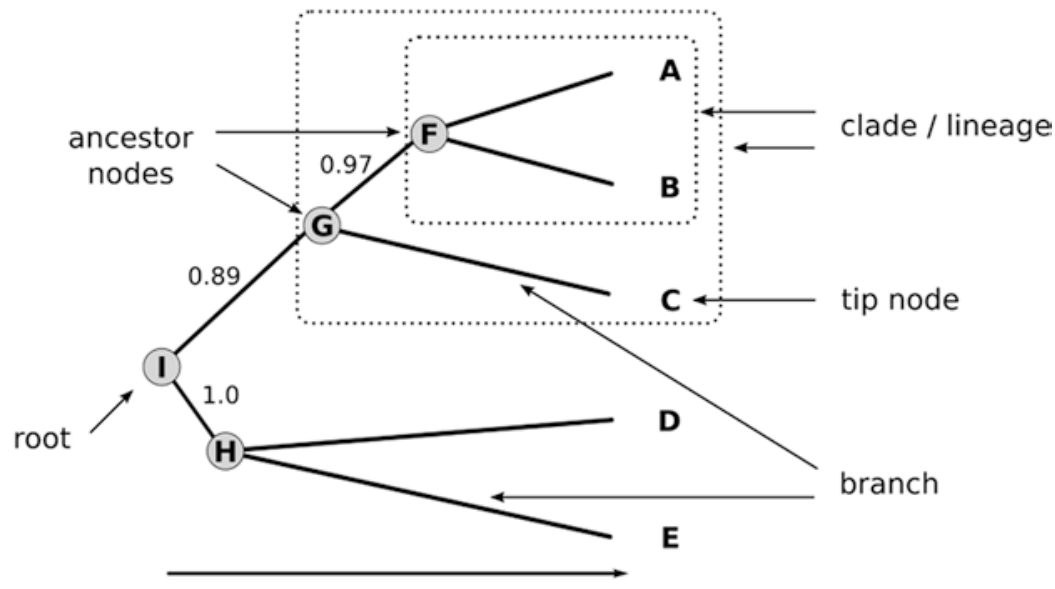

a

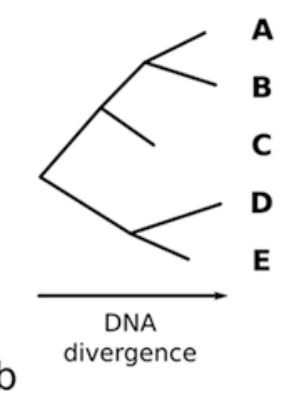

divergence time
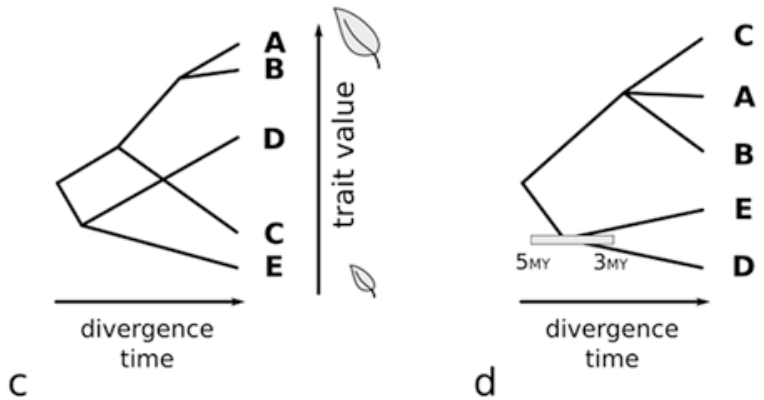

Fig. 7.1 Phylogenetic trees depict the inferred evolutionary relationships between species. (a) Clades (or lineages) are defined by a common ancestor and all of its descendants. Nodes are the branching points between descendants as well as tips, which are typically species. All nodes-tips and ancestors alike-share a common ancestor. The ancestral node from which all subsequent nodes of the tree descend is called the root. Confidence in the evolutionary relationships is shown above internal branches (maximum possible is 1). (b) Branch lengths (here shown along the $x$-axis) may represent divergence times, number of generations, or amount of molecular divergence. (c) In some cases the $y$-axis is used to display information about a quantitative trait—such as leaf sizein a tree known as a phenogram or a traitigram. (d) Unresolved relationships can be represented as three or more descendants stemming from the same ancestor, which is known as a polytomy. Uncertainty in divergence times are generally depicted with error bars at the internal nodes, if indicated at all

among species and lineages is also critical for making meaningful inferences about evolution. As we will discuss in the next section, estimates of the pace of trait evolution depends on the amount of change in a trait that occurs over a unit of time.

There are today several resources to help generate a good phylogenetic tree for a set of species. A common approach is to trim the whole plant tree of life-taken from the Open Tree of Life (Hinchliff et al. 2015) or Phylomatic (Webb and Donoghue 2005), for example - to the set of species of interest. A second option is 
to reconstruct the phylogeny from scratch using DNA sequences and then by timecalibrating the tree using fossil information and molecular clock models. Tree reconstruction is tricky and laborious, but there are many tools that can help (e.g., Antonelli et al. 2016; Pearse and Purvis 2013). Cobbling together a phylogenetic tree by manually assembling branches is not recommended for analysis of spectra or other traits.

Finally, as seen in the previous section, phylogenies are estimates, and systematists have means of assessing uncertainty in their topology and their branch lengths, which are together referred to as phylogenetic uncertainty. For example, the divergence between two lineages may have a mean of 20 million years and a confidence interval or $95 \%$ highest posterior density of 18-22 million years. That uncertainty can (and should) be carried over to downstream statistical analyses.

\subsection{The Evolution of Quantitative Traits}

The study of evolution is fundamentally concerned with describing how organisms change through time and with understanding the processes driving change. Evolutionary change, however, can be thought about at different phylogenetic and temporal scales. Because we are interested in understanding spectra in light of phylogenies, we will not discuss microevolutionary processes that occur at the population level such as genetic drift and natural selection. Instead, we will focus on describing macroevolution and how traits - such as leaf structure and chemical composition—change across entire lineages over long timescales (usually millions of years).

\subsubsection{Macroevolutionary Models of Trait Evolution}

Macroevolutionary models of trait evolution describe the long-term consequences of short timescale evolution. At any given time step, a trait value can increase or decrease due to mechanisms like selection, drift, and migration. For example, the reflectance in one spectral region may decrease due to selection for higher levels of a particular pigment, while reflectance in another spectral region may decrease due to a random change in leaf hair density. Many such changes occur over long evolutionary time in each lineage.

\subsubsection{Brownian Motion}

Most models for evolution of quantitative traits leverage the central limit theorem from statistics, which states that the sum of many random changes leads to a normal distribution. Because trait evolution at macroevolutionary scales integrates over 
many random changes in trait values (due to varied processes), it may be described by a normal distribution. This model of evolution is known as Brownian motion (Felsenstein 1985). The pace at which those changes accumulate is at the core of what we call the rate of evolution, and it is captured by the variance of the normal distribution (whose mean is the trait value at the root).

When lineages split, they start out with the same trait value and then diverge independently. It is easy then to see that the expected amount of trait variation between lineages depends on both the rate of evolution and on the divergence time. This leads to the expectation that trait values should be on average more similar among closely related taxa-which had little time to diverge- than among distantly related taxa. Such expectation is at the core of the concept of phylogenetic signal (see Sect. 7.3.2) and the idea that phylogenetic relatedness can be used as a proxy for functional similarity (Webb et al. 2002), particularly when integrating across a large number of traits (Cavender-Bares et al. 2009).

\subsubsection{Ornstein-Uhlenbeck}

With Brownian motion, an increase or decrease in a trait is equally likely, regardless of the current value of a trait (Fig. 7.2a). However, it could be more realistic to think of a trait as being pulled toward some optimum (or, similarly but not quite the same, away from extreme values). This force or "pull" could be due to many processes: it is often considered to be a pull toward some evolutionary optimum due to natural selection, but it could instead result from a bias in mutation toward a particular trait value, repulsion from extremes, or other factors that lead to a pattern that resembles a pull toward an optimum. The placement of the optimum, the strength of the pull, and the basic underlying rate of evolution are all parameters of this model, which is known as an Ornstein-Uhlenbeck process (Butler and King 2004). The degree of the pull toward the optimum is analogous to the strength of a rubber band linking
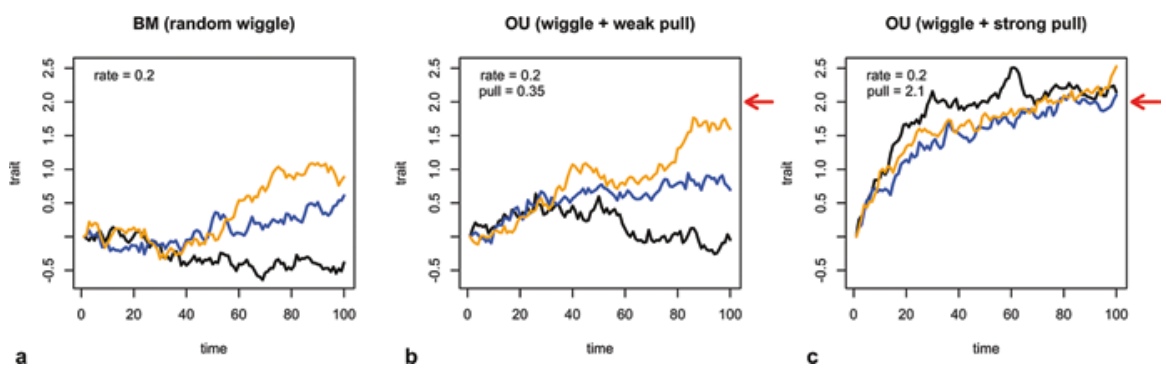

Fig. 7.2 Three independent realizations of the Brownian motion (BM) and Ornstein-Uhlenbeck (OU) processes. (a) In a BM model, trait values are equally likely to increase or decrease at each time step. (b, c) In contrast, traits in an OU model are more likely to move toward an optimum (represented by the red arrows). (b) When the evolutionary pull is weak, traits move slowly toward their optimum. (c) When the pull is strong, however, traits converge quickly toward their optimum 
the evolving trait on one end and the optimum trait value on the other end. A weak rubber band will provide enough slack for the trait to wiggle around the optimum (Fig. 7.2b), whereas a strong rubber band will keep the evolving trait close to the optimum (Fig. 7.2c). The strength of the rubber band also affects how quickly the trait is pulled toward its optimum (Fig. 7.2b,c). The time a trait is expected to take to get halfway to the optimum is called the phylogenetic half-life, and this is an alternative way to think about the strength of the evolutionary pull.

Variation in traits within species, populations, and even individuals may result from responses to environmental conditions or have a genetic basis. Until recently, phylogenetic comparative methods largely ignored intraspecific variation and used species means instead. Ives et al. (2007) and Felsenstein (2008) devised methods to account for within species variation, which typically enters the model as the standard errors about the mean trait value of each species.

\subsubsection{Phylogenetic Signal}

Phylogenetic signal can be thought as the degree to which closely related species resemble each other. Two different metrics have been widely used to assess phylogenetic signal: Pagel's lambda (Pagel 1999) and Blomberg's K (Blomberg et al. 2003).

\subsubsection{Pagel's Lambda}

Pagel's lambda is a scalar for the correlation between the phylogenetic similarity matrix and the trait matrix. It has the effect of shrinking the internal branches (as opposed to the branches that lead to the tips) of a phylogeny, thereby reducing the expected species correlation due to shared evolutionary history (Fig. 7.3a-d). A lambda value of 0 indicates that trait correlations between species are independent from evolutionary history (Fig. 7.3d), whereas a lambda of 1 suggests that trait correlations are equal to the species correlation imposed by their shared evolutionary history (Fig. 7.3a), assuming a Brownian motion model of evolution.

\subsubsection{Blomberg's $K$}

Blomberg's $K$ measures the degree to which trait variance lies within clades versus among clades. Brownian motion is used as an expectation. $K$ values greater than 1 indicate that there is more variance among clades than expected by Brownian motion (Fig. 7.3e), while $K$ values smaller than 1 imply that more variance is found within clades than expected under a Brownian motion model (Fig. 7.3f).

It is important to note that both Pagel's lambda and Blomberg's $K$ are treewide metrics, meaning that they do not explicitly account for the heterogeneity in trait values among lineages. For example, an estimate of low phylogenetic signal in fruit 
Fig. 7.3 This is how phylogenetic signal is inferred. (a-d) Pagel's lambda is equivalent to scaling the internal branches of the phylogeny, which reduces the expected covariance between species due to evolutionary history. (e, f) Blomberg's $K$ measures phylogenetic signal by estimating the degree of variation between and within clades. (e) $K$ value is high when most trait variation is found between clades instead of within them. (f) $K$ values are low when trait variation is mostly within clades
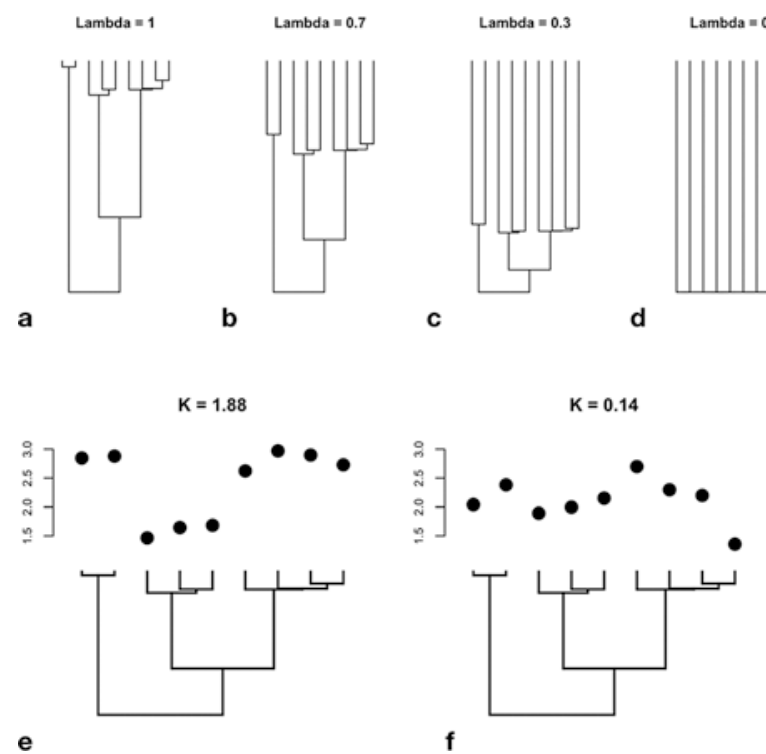

shape across all flowering plants does not imply the lack of phylogenetic signal in fruit shape within the oaks. Therefore, assessments of phylogenetic signal should be seen as indicators that are contingent on the scale of analysis and the particular species sampled instead of as general, hard truths.

It is also important to recognize that every calculation of the phylogenetic signal of a trait involves fitting an evolutionary model that comes with a series of assumptions. For example, most procedures to estimate phylogenetic signal using Blomberg's $K$ are based on a single-rate Brownian motion model; using and reporting the measure of phylogenetic signal implicitly requires accepting the Brownian assumptions.

\subsection{Evolution and Spectra}

Chemical and structural leaf attributes that underlie plant spectra evolve through time. Because leaf spectra integrate over these evolved leaf attributes, they can carry information about phylogenetic relationships and leaf evolution. Given this, how would one go about analyzing spectra in a phylogenetic context?

One approach is to subject the spectra directly to an evolutionary analysis, essentially taking reflectance values at different bands across the spectrum to be a set of “traits." For example, McManus et al. (2016) estimated Pagel's lambda on spectra from Amazonian plants, assuming each band to be an independent trait. CavenderBares et al. (2016) used principal component analysis (PCA) to reduce the dimensionality of the spectral data before estimating phylogenetic signal on the resulting principal component axes using Blomberg's $K$. 
a

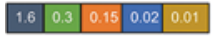

Leaf layers, Chlorophyll,

Carotenoids, H2O, LMA

\section{Ancestral}

PROSPECT

parameter values

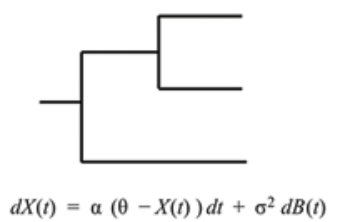

Known phylogenetic tree and model of evolution

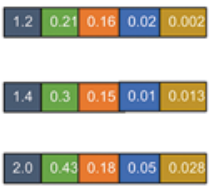

Evolved PROSPECT parameters
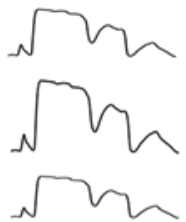

Leaf spectra evolved under a known tree and evolutionary regime

Fig. 7.4 Integration of trait evolution and leaf spectral models enables estimation of evolutionary parameters from spectra and simulation of leaf spectra along a phylogeny. Ancestral leaf attributes evolve along a phylogenetic tree under a given evolutionary regime, generating the current leaf attributes that underlie spectra. From the evolved leaf attributes, radiative transfer models (RTMs) such as PROSPECT estimate spectra that carry the signature of the phylogeny

Fitting evolutionary models directly to spectra can be useful for identifying promising associations between phylogenetic history and plant spectral signatures. However, this approach is largely devoid of mechanism and does not allow us to verify that our inferences are biologically meaningful.

Another approach to integrate phylogenies and leaf spectra is to explicitly model the evolution of structural and chemical traits that underlie the spectrum. This approach matches more closely the reality of biology by acknowledging that any signal of evolution found in the spectra is an emerging property of the evolutionary dynamics of leaf traits (see Sect. 7.5.3). This idea can be implemented by coupling the models of trait evolution described in the previous section with leaf radiative transfer models (Fig. 7.4) that predict spectral profiles from a small set of leaf attributes (see Martin, Chap. 5; Ustin and Jacquemoud, Chap. 14).

This framework can be used in several ways. For example, we can simulate what leaf spectra would look like given a certain evolutionary model and phylogenetic tree (Sect. 7.4.1). Alternatively, given a phylogeny and a spectral data set, we can infer what ancestral spectra or ancestral traits were like if we assume a certain model of evolution. Finally, given a spectrum from an unknown plant, we could estimate how that plant is related to other plants (Sect. 7.4.3).

\subsubsection{Simulating Leaf Spectra Under Different Evolutionary Regimes}

A model that describes the evolution of leaf spectra mediated by the evolution of leaf traits enables us to simulate spectral data in a phylogenetically explicit way. This allows us to forecast how different evolutionary scenarios would affect the shape and diversity of spectral profiles we observe. For example, Fig. 7.5 shows how the different scenarios for the evolution of leaf structure- the number of layers parameter $(\mathrm{N})$ 

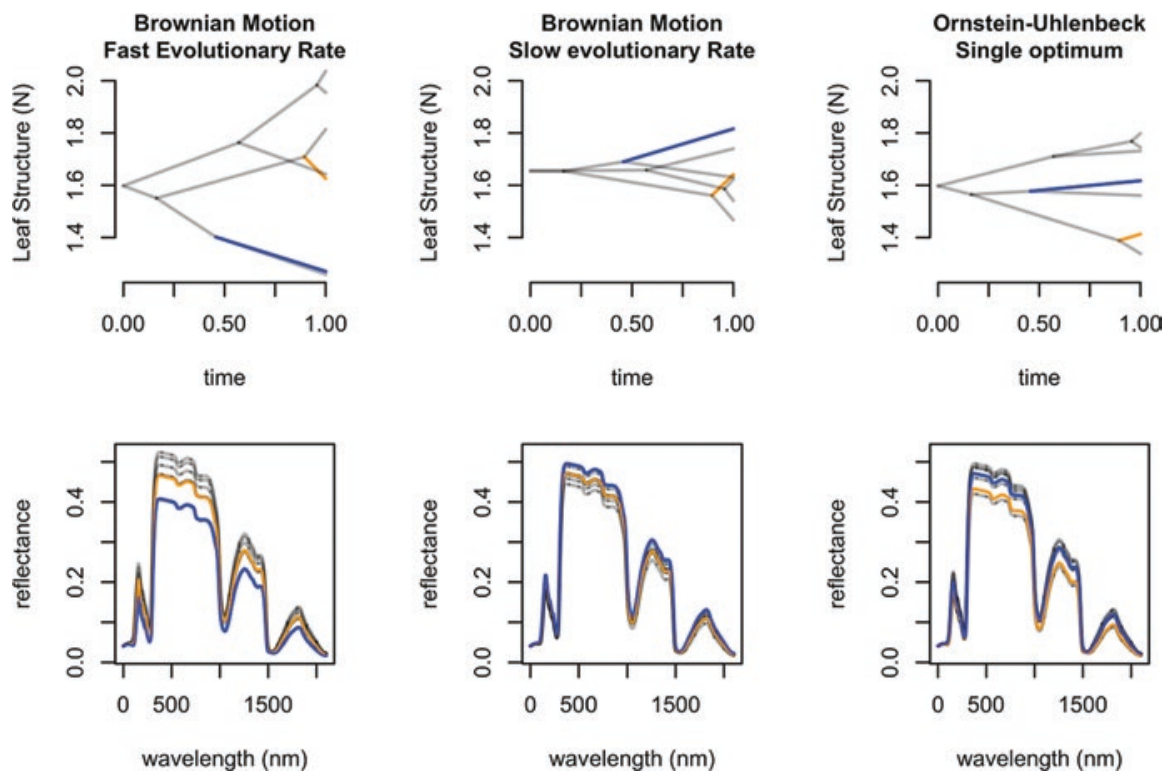

Fig. 7.5 Evolution of leaf structure under different evolutionary scenarios and consequences for leaf spectra. Top row depicts evolution according to an unbounded Brownian motion model at two different rates and according to an Ornstein-Uhlenbeck process. The bottom row shows spectra estimated with the PROSPECT5 model, where all leaf attributes evolved under the same model except for leaf structure, which evolved under the three scenarios outlined above

in PROSPECT5 - result in different amounts of trait variability. A fast Brownian rate (top left, Fig. 7.5) results in higher trait variation than a slow Brownian rate (top center, Fig. 7.5). Evolution under an Ornstein-Uhlenbeck model also results in less variation than the fast Brownian model even though their rates of evolution are the same (top right, Fig. 7.5). The trait values shaped by evolution have a noticeable effect on the spectral profiles of those lineages (bottom panels, Fig. 7.5).

\subsubsection{Making Evolutionary Inferences from Leaf Spectra}

Integrating spectra and phylogenies raises the exciting prospect of leveraging spectra to estimate aspects of the evolutionary process and test hypotheses.

Some questions may be about evolutionary patterns in the spectra themselves. Those include investigations about phylogenetic signal or rates of evolution across the spectrum. For example, Cavender-Bares et al. (2016) and McManus et al. (2016) investigated how much phylogenetic signal is present in leaf spectra. Meireles et al. (in review) estimated how rates of evolution varied across the leaf spectrum of seed plants. Now, because we are interested in biology, evolutionary inference made at the spectral level will often need to be interpreted a posteriori. 
Interpreting results correctly may pose some challenges, however. Who guarantees that the high rates of evolution in a particular spectral band really means that a certain trait is evolving at a fast pace? A potentially better approach is to infer traits from spectra first using either statistical (e.g., partial least squares regression) or RTM inversions (e.g., PROSPECT) and then study the evolution of those traits (see Serbin and Townsend, Chap. 3).

We can test hypotheses about how evolution affects leaf spectra because we can calculate the likelihood of spectral data being generated by different models of evolution, which can be compared to each other using a goodness of fit metric such as Akaike information criterion (AIC; Burnham and Anderson 2002). We foresee numerous interesting hypotheses being tested using this type of approach, especially related to evolutionary rates and convergent evolution.

Here is a hypothetical but realistic example: We could hypothesize that plant lineages that shift from sunny to shade habitats see an increase in their leaf chlorophyll content from 20 to $60 \mathrm{ug} / \mathrm{cm}^{2}$, that is, they have a new chlorophyll content optimum, and that should be reflected in their spectra (Fig. 7.6). We used the predictive approach established in the previous subsection to simulate leaf spectra under that evolutionary scenario, which highlights the disparity in reflectance in the visible spectrum between sun and understory plants. We can then fit various models of evolution to the spectra (including one- and two-rate Brownian motion as well as a one-optimum Ornstein-Uhlenbeck and a two-optimum Ornstein-Uhlenbeck, the model under which the data were simulated), calculate their AIC, and compare models using AIC weights (Burnham and Anderson 2002), as shown in Fig. 7.6. In this simulated scenario, we find that indeed the best fit comes from having two different optima in the spectra correlating with chlorophyll content. However, in real data, we might find that there is a difference, but only in bands correlating with lignin content in leaves (which could reflect different herbivore or structural pressure); that there is a difference in optimum but that understory plants are much more constrained toward their optimum than plants from sunnier habitats; or that there is a change but it happens over longer time periods than we expect.

\subsubsection{Leaf Spectra, Biodiversity Detection, and Evolution}

As other chapters discuss, one approach to assessing biodiversity from plant spectra is to use spectral indices that correlate with species richness (Gamon et al., Chap. 16). Another approach is to use classification models (Clark et al. 2005; Asner and Martin 2011; Serbin and Townsend, Chap. 3). Using an empirical example within a single lineage (the oaks, genus Quercus), there is enough information in the spectra of leaves to significantly differentiate populations within a single species (Quercus oleoides) and assign them to the correct population most of the time. Different species can be correctly classified with even greater accuracy, and the four major oak clades in the example can be identified with very high accuracy (Cavender-Bares et al. 2016). 
A
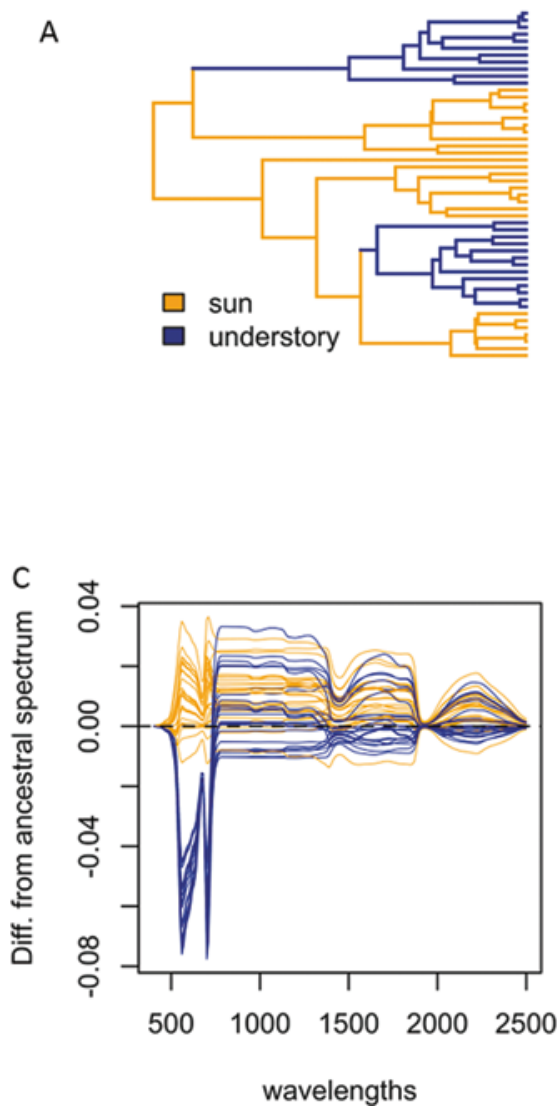

B

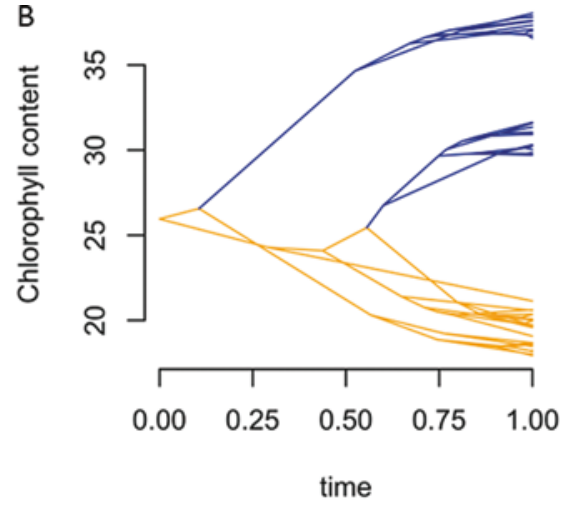

D

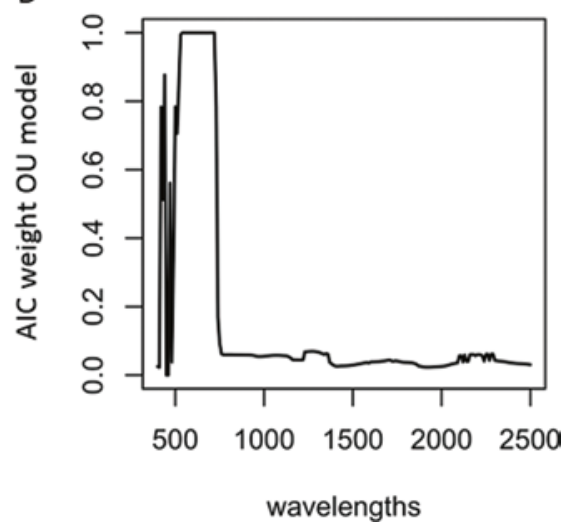

Fig. 7.6 Simulation of the evolution of chlorophyll content under a multiple optima OrnsteinUhlenbeck model. (a, b) Macroevolutionary shifts from sun exposed to understory habitats (a) result in chlorophyll content being pulled toward different optima in different lineages (b). (c) Differences between the evolved spectra and the ancestral spectrum highlight the effect of chlorophyll evolution on the visible region of the spectrum. (d) We can use AIC to calculate how well various models of evolution, including the true multiple optima Ornstein-Uhlenbeck model, describe evolution across the spectrum. AIC weights suggest that the multiple optima OrnsteinUhlenbeck model is preferred in the visible regions and nowhere else, which matches how the data were simulated

Evolutionarily-explicit diversity detection approaches could have enormous potential even when species cannot be identified. Biodiversity encompasses, among other things, which branches of the tree of life are found in an area how much evolutionary history that represents. Because plant spectral profiles can carry information about evolutionary history, they can be leveraged to assess the diversity of lineages instead of (or in addition to) the diversity in species or function. There are key conceptual advantages of taking this approach.

First, we can estimate lineage diversity at different phylogenetic scales when species-level detection performs poorly. As suggested in Fig. 7.7, leaf spectral 
Fig. 7.7 Classification accuracy for different diversity levels of Quercus:

(1) populations with Quercus oleoides, (2) 33 oak species, and (3) 4 clades of the genus Quercus. Accuracy was estimated from 300 independent PLS-DA iterations and summarized using Cohen's kappa. (Redrawn from CavenderBares et al. (2016))

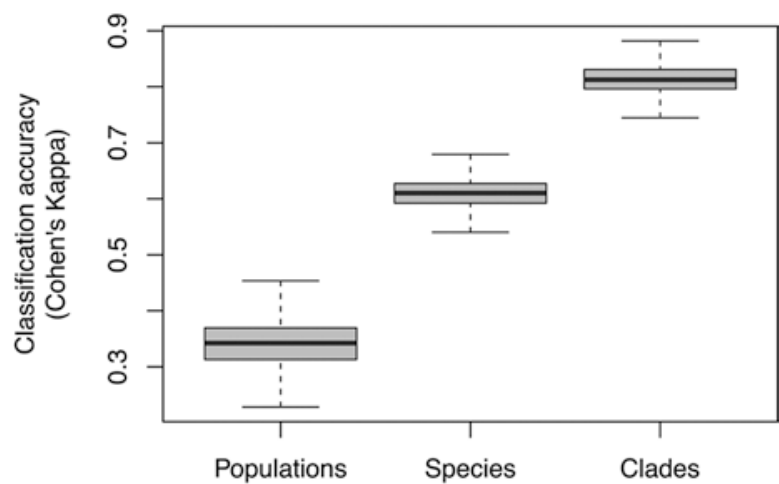

Diversity level

information can more accurately identify broad oak clades (kappa 0.81) than species (kappa 0.61) and than population within a species (kappa 0.34). It is thus possible that classification models can detect broad clades more accurately than they can detect very young clades or species.

Second, we know that species definitions change over time and that many species in hyperdiverse ecosystems are still unknown to science. How can we classify species that we do not yet know about? Using evolutionary models to estimate where an unknown spectrum belongs on the tree of life reduces the need for labeling, because it can reveal the taxa that the unknown sample is related to. This provides a means to estimate the phylogenetic diversity of a site even without species identities. The models of evolution described above should allow us to calculate the probability that the unknown spectrum belongs to different parts of the tree assuming that we know the correct evolutionary model and its parameter values. Developing the framework to achieve this would require filling in many gaps and detecting species at appropriate spatial resolutions. It also would require trusting many assumptions that go into evolutionary models, because we know that as we go deeper in phylogenetic time and evolutionary history, these models become increasingly complex (see Sect. 7.5.2).

\subsubsection{Diversity Detection at Large Scales: Challenges and Ways Forward}

The fact that spectra are tightly coupled with evolutionary history helps explain why hyperspectral data can be used for accurate taxonomic classification. It also provides a basis for using remotely sensed hyperspectral data for biodiversity composition monitoring.

Using RS hyperspectral data for biodiversity detection requires moving from the leaf level to the whole canopy level (Serbin and Townsend, Chap. 3; Martin, Chap. 5; Gamon et al., Chap. 16). We expect that canopy spectra, like leaf spectra, will show 
tight coupling to phylogenetic information. Branching architecture, leaf angles, and other structural traits of plants that contribute to spectral signals at the canopy level are themselves evolved traits and are potentially phylogenetically conserved. To the extent that remotely sensed hyperspectral data can capture the spectral profiles of individual canopies (Gamon et al., Chap. 16), hyperspectral data should be capable of detecting and identifying species (Morsdorf et al., Chap. 4) and lineages, following the logic presented above for leaves. Such an effort would require assembling vast libraries of spectral information across the plant tree of life for a given region of interest and comparing spectra obtained through remote sensing to those libraries.

Developing accurate classification models as the number of species and clades grow can be challenging. For example, Fig. 7.8 shows randomly assembled communities with different species diversity levels, where species spectra were simulated using PROSPECT5. As the number of species grows, the ability of a PLS-DA classification model to correctly classify species decreases.

This classification problem can be simplified by circumscribing the possible species pool. This could be done by estimating the potential pool of species or clades in a region based on other biodiversity monitoring and prediction approaches, including herbarium records, plant inventories or other types of in-situ data collection, and habitat suitability predictions (Pinto-Ledezma and Cavender-Bares, Chap. 9). Combining classification methods using hyperspectral data with prediction of species pools at regional scales across the globe could allow global plant compo-

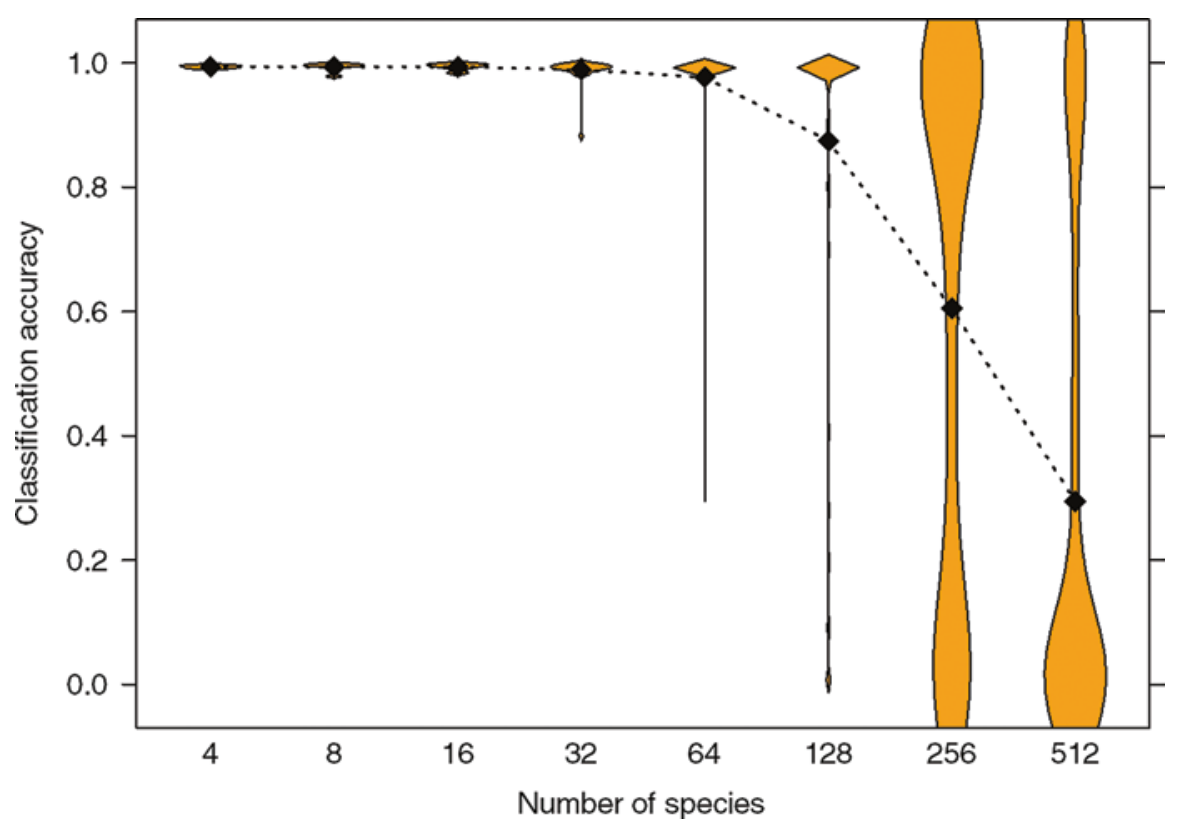

Fig. 7.8 Species classification accuracy of a PLS-DA model in simulated communities with different species richness. Spectra for each species were simulated using PROSPECT5, and communities with different diversity levels were randomly assembled 
sition information to be detected when the spatial resolution of the data is sufficient to capture individuals.

\subsection{Cautionary Notes}

The integration of leaf spectra and phylogenies can provide breakthroughs in how we detect biodiversity, explain how spectral variation between species and lineages comes to be, and make inferences about the evolution of leaves. We should, nevertheless, be aware of the limitations inherent in making inferences about the deep past, be mindful of the sampling requirements and statistical assumptions of our analyses, and be careful to interpret our findings in a biologically meaningful way.

\subsubsection{Is the Sampling Adequate for Making Evolutionary Inferences?}

Inferences about the evolutionary process or that rely heavily on it-such as the degree of phylogenetic signal or the pace of evolution-are dependent on how well a lineage has been sampled. Evolutionary biologists usually target a particular lineage and strive to include in their analysis as many close relatives as possible regardless of their location. Ecologists, on the other hand, tend to focus on a specific geographic area of interest and end up sampling whatever species are there. This likely results in very severe undersampling of the total phylogenetic diversity represented by the particular species pool. For example, the 20 seed plants at a study site belong to a clade that has about 300,000 species, harbors incredible morphological and physiological diversity, and goes back 350 million years. Evolutionary analyses of undersampled will very likely yield poor estimates of the evolutionary parameters: The species in that area can tolerate a subset of all the climate conditions other seed plants can handle, for example.

In addition, ecological processes themselves can lead to bias in estimates of evolutionary parameters. For example, extremely arid conditions may act as an environmental filter that curbs colonization by species with low leaf water content, reducing the amount of variability in leaf succulence. As a consequence, estimates of the rate of evolution of leaf succulence based on species found in that hyper-arid community may be artificially low.

These caveats should be kept in mind when analyzing spectra in an evolutionary context. Finding that certain spectral regions have high phylogenetic signal in a large forest plot does not necessarily mean that those regions are truly phylogenetically conserved. 


\subsubsection{The More of the Tree of Life That Is Sampled, the More Complex Models Will (or Should) Be}

Most of the models of evolution and phylogenetic signal statistics we saw here are actually rather simple. For example, a Brownian motion model has two parameters, the trait value at the root (mean) and the rate of evolution (variance). The single-rate Brownian motion model may reasonably describe the evolution of leaf water content in dogwoods (Cornus), but it would probably do a terrible job if you were analyzing all flowering plants because of the sheer heterogeneity and diversity that they possess (Felsenstein 2008; O’Meara 2012; Cornwell et al. 2014).

There is a trade-off: the most realistic model would have a different set of parameters at every time point on every branch but would have far more parameters to estimate than the data could support; a simple model of one set of parameters across all the time periods and species examined is clearly unrealistic. Most applications have used the simplest approach, but there are ways to allow for more complex models. Some of them test a priori hypotheses about heterogeneity in models of evolution: Biologists propose particular models linking sets of parameters on different parts of the tree (e.g., gymnosperms and angiosperms having different rates of evolution), and then the methods select between the possible models (Butler and King 2004; O'Meara et al. 2006). There are also methods that can automatically search across possible mappings to find the ones that fit best (Uyeda and Harmon 2014). In the case of multiple characters, such as reflectance at different wavelengths of light, there is also the question of whether different characters are evolving under the same or different models, and there are models to test that, as well (Adams and Otárola-Castillo 2013).

Early attempts to analyze spectra in an evolutionary context (Cavender-Bares et al. 2016; McManus et al. 2016; Meireles et al. in review) have used models that are maximally simple for each character (a single model applying for all taxa and times) and are nearly maximally complex between characters (each trait evolves independently of all others on the same common tree). Those approaches are computationally cheap but are at odds with our understanding of biology (i.e., models of evolution do vary among lineages) and physics (i.e., spectral bands do covary). Other ways of segregating complexity, such as models that incorporate heterogeneity among lineages and account for the covariance among spectral bands, remain potentially more fruitful ways of examining the diversity in leaf spectra.

\subsubsection{Spectra Do not Evolve*, Leaves Do!}

$\{$ *except when they do $\}$

One could estimate the pace of evolution of the beaks of Darwin's finches from their photographs. But the photographs didn't evolve. Leaf spectra do capture many different aspects of the complex phenotype, and, we have seen in this chapter, each 
band of a spectrum can be analyzed as a trait in an evolutionary model. This does not necessarily mean that spectra themselves are traits nor that they themselves evolve. For example, there is no reason for evolution to favor lower reflectance at $660 \mathrm{~nm}$. However, there may be biological reasons for natural selection to favor higher amounts of chlorophyll a in a leaf, which happens to absorb light at $660 \mathrm{~nm}$. Terminology such as "evolution of spectra" or "spectral niches" may be efficient communication shortcuts but can also cause confusion. They may make it all too easy to lose sight of the biological mechanisms behind the observed phenomena.

Advances in analyzing spectral data in light of evolution will require keeping mechanisms in mind. That said, phylogenetic inference on spectra can be used as a discovery tool. Consistently finding high rates evolution in a spectral region not associated with a known function should trigger further investigation. Moreover, mechanistic thinking may end up proving us wrong and show that spectra in fact evolve (at least some regions). For example, increased leaf reflectance that prevents leaf overheating could be favored by evolution. In such a situation, high reflectance would result from "real" traits—such as bright hairs, cuticles, and waxes_but one can argue that there is biological meaning in the evolution of reflectance itself in this case.

\subsubsection{Ignore Phylogeny at Your Peril}

Phylogeny adds complexity to an analysis but has benefits in new insights (estimating ancestral leaf spectra, helping to go from observations to traits, and more). However, it can be tempting to analyze data on multiple species without accounting for shared evolutionary history. The problem with methods that ignore the phylogeny, such as partial least squares regression, is that they assume that species are independent data points. They are not! There is thus the risk of "overcounting" some parts of the tree of life: for example, if one wants to develop a model for all plants, and one has five oak species, a ginkgo, a pine, and a magnolia, the final model will essentially be an oak model with some deviations. However, the five oaks have shared much of their evolutionary history and so do not represent five independent instances of evolution. Phylogenies can be included into such analyses, and their importance appropriately scaled (in some cases, they will not affect results, but this is only knowable once the tree is used), and make results far more robust.

\subsection{Moving Forward}

The integration of leaf spectra and phylogenies using evolutionary models is still in its infancy. Phylogenetic models have the potential to unlock what drives evolution of the traits leading to different spectra. Spectra may have the potential, combined with phylogenies, to help identify species from afar, and even contain phylogenetic information themselves. 
Acknowledgments We are grateful to Shan Kothari, Laura Williams, and Jesús Pinto-Ledezma for their feedback on an early version of this manuscript. We are also grateful to the National Institute for Mathematical and Biological Synthesis (NIMBioS) for funding the "Remotely Sensing Biodiversity" working group.

\section{References}

Adams DC, Otárola-Castillo E (2013) Geomorph: an R package for the collection and analysis of geometric morphometric shape data. Methods Ecol Evol 4(4):393-399

Antonelli A, Hettling H, Condamine FL, Vos K, Henrik Nilsson R, Sanderson MJ, Sauquet H et al (2016) Toward a self-updating platform for estimating rates of speciation and migration, ages, and relationships of taxa. Syst Biol 66(2):152-166. Oxford University Press: syw066

Asner G, Martin R (2011) Canopy phylogenetic, chemical and spectral assembly in a lowland Amazonian forest. New Phytol 189:999-1012

Blomberg SP, Garland T, Ives AR (2003) Testing for phylogenetic signal in comparative data: behavioral traits are more labile. Evolution 57(4):717-745

Burnham KP, Anderson DR (2002) Model selection and multimodel inference: a practical information-theoretic. Springer, New York, NY

Butler MA, King AA (2004) Phylogenetic comparative analysis: a modeling approach for adaptive evolution. Am Nat 164(6):683-695. The University of Chicago Press

Cavender-Bares J, Kozak KH, Fine PV, Kembel SW (2009) The merging of community ecology and phylogenetic biology. Ecol Lett 12:693-715

Cavender-Bares J, Meireles JE, Couture JJ, Kaproth MA, Kingdon CC, Singh A, Serbin SP et al (2016) Associations of leaf spectra with genetic and phylogenetic variation in oaks: prospects for remote detection of biodiversity. Remote Sens 8(3):221

Clark M, Roberts D, Clark D (2005) Hyperspectral discrimination of tropical rain 10 forest tree species at leaf to crown scales. Remote Sens Environ 96:375-398

Cornwell WK, Westoby M, Falster DS, FitzJohn RG, O’Meara BC, Pennell MW, McGlinn DJ et al (2014) Functional distinctiveness of major plant lineages. Edited by Amy Austin. J Ecol 102(2):345-356

Felsenstein J (1985) Phylogenies and the comparative method. Am Nat 125(1):1-15

Felsenstein J (2008) Comparative methods with sampling error and within-species variation: contrasts revisited and revised. Am Nat 171(6):713-725

Hinchliff CE, Smith SA, Allman JF, Gordon Burleigh J, Chaudhary R, Coghill LM, Crandall KA et al (2015) Synthesis of phylogeny and taxonomy into a comprehensive tree of life. Proc Natl Acad Sci U S A 112(41):12764-12769

Ives AR, Midford PE, Garland T (2007) Within-species variation and measurement error in phylogenetic comparative methods. Syst Biol 56(2):252-270

McManus K, Asner GP, Martin RE, Dexter KG, John Kress W, Field C (2016) Phylogenetic structure of foliar spectral traits in tropical forest canopies. Remote Sens 8(3):196

O'Meara BC (2012) Evolutionary inferences from phylogenies: a review of methods. Ann Rev Ecol Evol 43:267-285

O’Meara BC, Ané C, Sanderson MJ, Wainwright PC (2006) Testing for different rates of continuous trait evolution using likelihood. Evolution 60(5):922-933

Pagel M (1999) Inferring the historical patterns of biological evolution. Nature 401(6756):877-884

Pearse WD, Purvis A (2013) PhyloGenerator: an automated phylogeny generation tool for ecologists. Edited by Emmanuel Paradis. Methods Ecol Evol 4(7):692-698 
Uyeda JC, Harmon LJ (2014) A novel Bayesian method for inferring and interpreting the dynamics of adaptive landscapes from phylogenetic comparative data. Syst Biol 63(6):902-918

Webb CO, Ackerly DD, McPeek MA, Donoghue MJ (2002) Phylogenies and Community Ecology. Annu Rev Ecol Syst 33:475-505

Webb CO, Donoghue MJ (2005) Phylomatic: tree assembly for applied phylogenetics. Mol Ecol Notes 5(1):181-183

Open Access This chapter is licensed under the terms of the Creative Commons Attribution 4.0 International License (http://creativecommons.org/licenses/by/4.0/), which permits use, sharing, adaptation, distribution and reproduction in any medium or format, as long as you give appropriate credit to the original author(s) and the source, provide a link to the Creative Commons license and indicate if changes were made.

The images or other third party material in this chapter are included in the chapter's Creative Commons license, unless indicated otherwise in a credit line to the material. If material is not included in the chapter's Creative Commons license and your intended use is not permitted by statutory regulation or exceeds the permitted use, you will need to obtain permission directly from the copyright holder.

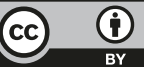

